\title{
EXISTENCE IN THE LARGE OF PARALLELISM HOMOMORPHISMS
}

\author{
BY \\ ROBERT HERMANN(1)
}

1. Introduction. All manifolds, tensor-fields, curves, maps, etc., will be of differentiability class $C^{\infty}$ unless mentioned otherwise. All manifolds will be paracompact and connected. Let $M$ be a manifold of dimension $n$. $(1 \leqq i, j, \cdots \leqq n$; summation convention.) An everywhere linearly independent set of 1-differential forms, $\left(\omega_{i}\right)$, on $M$ defines an absolute parallelism for $M$. We will consider one such parallelism as fixed on $M$. Let $M^{\prime}$ be another manifold, with a set of 1-forms $\left(\omega_{i}^{\prime}\right)$, not necessarily defining a parallelism. Our problem: When is there a map $\phi: M^{\prime} \rightarrow M$ such that $\phi^{*}\left(\omega_{i}\right)=\omega_{i}^{\prime}$ ? (If $\omega$ is a differential form on $M, \phi^{*}(\omega)$ is the form on $M^{\prime}$ induced by $\phi$, denoted by $\delta \phi(\omega)$ in [4].) Suppose that:

$$
d \omega_{i}=c_{j k i} \omega_{j} \wedge \omega_{k}
$$

The $c_{j k i}$ are the first order invariants of the parallelism on $M$. Define the second, third, $\cdots$ order invariants $c_{j k i, h}, c_{j k i, h_{1}, h_{2}}, \cdots$ as follows:

$$
d c_{j k i}=c_{j k i, h} \omega_{h}, \quad d c_{j k i, h_{1}}=c_{j k i, h_{1}, h_{2}} \omega_{h_{2}}, \cdots
$$

Suppose further that:

1.3. There are functions $c_{j k i}^{\prime}, c_{j k i, h}^{\prime}, \cdots$ on $M^{\prime}$ such that

$$
\begin{aligned}
& d \omega_{i}^{\prime}=c_{j k i}^{\prime} \omega_{j}^{\prime} \wedge \omega_{k}^{\prime}, \\
& d c_{j k i}^{\prime}=c_{j k i, h}^{\prime} \omega_{h}^{\prime}, \text { etc }
\end{aligned}
$$

Let $x_{0}$ (resp $\left.x_{0}^{\prime}\right)$ be a fixed point of $M\left(\operatorname{resp} M^{\prime}\right)$ and let $P\left(\operatorname{resp} P^{\prime}\right)$ be the space of $\left(C^{\infty}\right)$ curves of $M$ starting at $x_{0}$ (resp at $\left.x_{0}^{\prime}\right)$. If $\phi$ exists, with $\phi\left(x_{0}^{\prime}\right)=x_{0}$, it defines a map $\phi_{*}: P^{\prime} \rightarrow P$. The method of Ambrose and Hicks $[1 ; 9]$ is conversely to first construct $\phi_{*}$, then find the conditions that it pass to the quotient with respect to the natural projections $P^{\prime} \rightarrow M^{\prime}$ and $P \rightarrow M$. Usually, this involves some sort of completeness condition for $M$, a condition on the fundamental group of $M^{\prime}$, and a requirement that the corresponding first order invariants are the same along curves corresponding under $\phi_{*}$. Our aim is to build a bridge between this

Received by the editors June 23, 1961 and, in revised form, July 15, 1962.

(1) Lincoln Laboratory, Massachusetts Institute of Technology, operated with support from the U.S. Army, Navy and Air Force. 
work and the work of E. Cartan on the problem ([2, Volume 2, Part 2, p. 724] and $[3$, p. 316]), which at first sight has a purely local character. The main idea is to find further sufficient conditions that the corresponding invariants be the same along the corresponding curves.

We will now state our main result. Let $\Omega$ be the ring of $C^{\infty}$ real valued functions on $M$ generated by the functions $c_{j k i}, c_{j k i, h}, \cdots$. Suppose further that there is a manifold that we will denote by $M / \Omega$, and a maximal-rank mapping $\pi: M \rightarrow M / \Omega$ such that:

1.4. For $x \in M$, the fibre of $\pi$ through $x$ is the connected component containing $x$ of $\{y \in M: f(y)=f(x)$ for all $f \in \Omega\}$.

THEOREM 2. Suppose that 1.1-1.4 are satisfied, that $M^{\prime}$ is simply connected and that the parallelism defined by the $\left(\omega_{i}\right)$ on $M$ is complete in the sense to be defined below. A given $f \in \Omega$ is constant on the fibres of $\pi$, hence there is a function f on $M / \Omega$ such that $\pi^{*}(\mathbf{f})=f$. Suppose that there is a map $\psi: M^{\prime} \rightarrow M / \Omega$ such that

$$
\psi^{*}\left(\mathbf{c}_{j k i}\right)=c_{j k i}^{\prime}, \quad \psi^{*}\left(\mathbf{c}_{j k i, h}\right)=c_{j k i, h}^{\prime}, \cdots
$$

Then, there is a map $\phi: M^{\prime} \rightarrow M$ such that

$$
\pi \phi=\psi \text { and } \phi^{*}\left(\omega_{i}\right)=\omega_{i}^{\prime} .
$$

Finally, in $\S 4$, we apply these ideas to prove an extension to spaces with absolute parallelism of de Rham's theorem on reducibility of Riemannian manifolds [5]. The methods are different from de Rham's and can be extended to also give a new proof of his result. We sketch this proof in $\$ 5$.

2. Completeness and the existence of homomorphisms. Let $\sigma:[0, T] \rightarrow M$ be a curve in $M$. The functions $a_{i}(t)=\omega_{i}\left(\sigma^{\prime}(t)\right), 0 \leqq t \leqq T$, are called the first order invariants of the curve $\sigma .\left(\sigma^{\prime}(t)\right.$ is the tangent vector to $\sigma$ at $\sigma(t)$, an element of $M_{\sigma(t)}$, the tangent space to $M$ at $\sigma(t)$.) Since we also have $\sigma^{*}\left(\omega_{i}\right)=a_{i}(t) d t$, we see that:

2.1. The curve $t \rightarrow(\sigma(t), t)$ in $M \times[0, T]$ is the integral curve of a vector field $X$ on $M \times[0, T]$ with $d t(X)=1$ and $\omega_{i}(X)=a_{i}$. Hence, given functions $a_{i}(t)$ and a point $x_{0} \in M$, there is at most one curve $\sigma$, with $\sigma(0)=x_{0}$, whose first order invariants are $a_{i}$.

Such a $\sigma$ can, by the usual process of analytic continuation, be defined over a maximal subinterval $\left[0, t_{0}\right)$. If $\sigma$ exists over $[0, T]$ for every choice of $T$ and functions $a_{i}(t)$ and every initial point $x_{0}$, then the parallelisms on $M$ is said to be complete (in the sense of Ehresmann [6]). Those curves whose first order invariants are constants will be called the straight lines of the parallelism (i.e., they are the curves whose tangent vector field is self-parallel).

Note that completeness in the usual sense of the theory of affinely connected 
manifolds means straight line completeness, i.e., a piece of a straight line can be indefinitely extended. This idea of completeness, although serving admirably in Riemannian geometry, is probably too weak in non-Riemannian situations.

Proposition 2.1. Let $M$ and $M^{\prime}$ be manifolds, with basepoints $x_{0} \in M$ and $x_{0}^{\prime} \in M^{\prime}$. Suppose there are forms $\left(\omega_{i}\right)$ and $\left(\omega_{i}^{\prime}\right)$ on $M$ and $M^{\prime}$, with:

$$
d \omega_{i}=c_{j k i} \omega_{j} \wedge \omega_{k}, \quad d \omega_{i}^{\prime}=c_{j k i}^{\prime} \omega_{j}^{\prime} \wedge \omega_{k}^{\prime} .
$$

Suppose that the $\left(\omega_{i}\right)$ define an absolute parallelism for $M$ which is complete in the sense defined above. For every curve $\sigma:[0, T] \rightarrow M^{\prime}$ with $\sigma(0)=x_{0}^{\prime}$ define a curve $\sigma_{D}:[0, T] \rightarrow M$ such that $\sigma_{D}(0)=x_{0}$ and $\sigma_{D}^{*}\left(\omega_{i}\right)=\sigma^{*}\left(\omega_{i}^{\prime}\right), \sigma_{D}$ is called the development of $\sigma$ in $M$. If $M^{\prime}$ is simply connected, a sufficient condition that there exist a map $\phi: M^{\prime} \rightarrow M$ with $\phi\left(x_{0}^{\prime}\right)=x_{0}$ and $\phi^{*}\left(\omega_{i}\right)=\omega_{i}^{\prime}$ is that:

2.3. $c_{j k i}^{\prime}(\sigma(t))=c_{j k i}\left(\sigma_{D}(t)\right)$ for each curve $\sigma$ in $M$ beginning at $x_{0}$.

Proof. Let $\delta(s, t), 0 \leqq s, t \leqq 1$, be a homotopy $\left({ }^{2}\right)$ of curves in $M^{\prime}$, with $\delta(s, 0)=x_{0}^{\prime}$. Let $\delta_{D}(s, t)$ be the developed homotopy in $M$, i.e., $\delta_{D}(s, 0)=x_{0}$ and $t \rightarrow \delta_{D}(s, t)$ is the development in $M$ of the curve $t \rightarrow \delta(s, t)$. It is clear that $\delta_{D}$ is $C^{\infty}$, since it is obtained from $\delta$ by solving $C^{\infty}$ differential equations with $C^{\infty}$ initial conditions. Suppose that

2.4

$$
\delta_{D}^{*}\left(\omega_{i}\right)=A_{i}(s, t) d t+B_{i}(s, t) d s,
$$

$$
\delta^{*}\left(\omega_{i}^{\prime}\right)=A_{i}^{\prime} d t+B_{i}^{\prime} d s .
$$

Using 2.2, we have:

$$
\frac{\partial A_{i}}{\partial s}-\frac{\partial B_{i}}{\partial t}=\delta_{D}^{*}\left(c_{j k i}\right) A_{k} B_{j}
$$

2.5 .

$$
\frac{\partial A_{i}^{\prime}}{\partial s}-\frac{\partial B_{i}^{\prime}}{\partial t}=\delta^{*}\left(c_{j k i}^{\prime}\right) A_{k}^{\prime} B_{j}^{\prime} .
$$

Notice that: $A_{i}=A_{i}^{\prime}$. Hence, using 2.3,

$$
\frac{\partial l}{\partial t}\left(B_{i}-B_{i}^{\prime}\right)=\delta^{*}\left(c_{j k i}\right) A_{j}\left(B_{k}-B_{k}^{\prime}\right),
$$

i.e., $B_{i}-B_{i}^{\prime}$ with $s$ held fixed, satisfies a linear, homogeneous differential equation in $t$. The condition that $\delta_{D}(s, 0)=x_{0}, \delta(s, 0)=x_{0}^{\prime}$ implies that $B_{i}=B_{i}^{\prime}=0$ at $t=0$, i.e.,

2.7. $B_{i}=B_{i}^{\prime}$ identically.

(2) All homotopies will also be $C^{\infty}$. Recall that two $C^{\infty}$ curves that are continuously homotopic are $C^{\infty}$ homotopic $[14$, p. 25$]$. 
In particular, if $\delta$ is a homotopy with both endpoints held fixed, so is $\delta_{D^{\prime}}$ hence, since $M^{\prime}$ is simply connected there is a bonafide map $\phi: M^{\prime} \rightarrow M$ such that $\phi(\sigma)=\sigma_{D}$ for each curve $\sigma$ starting at $x_{0}^{\prime}$. That this map is $C^{\infty}$ again follows from the fact that it is obtained locally by solving $C^{\infty}$ differential equations. 2.7 now again implies that $\phi^{*}\left(\omega_{i}\right)=\omega_{i}^{\prime}$, since if $\delta$ is a homotopy in $M^{\prime}$ and $v^{\prime} \in M_{\delta(s, t)}^{\prime}$ (resp $v \in M_{\delta_{D}(s, t)}$ ) is the tangent vector to the curve $u \rightarrow \delta(u, t)$ (resp $\left.u \rightarrow \delta_{D}(u, t)\right)$ at $u=s$, then $B_{i}(s, t)=\omega_{i}(v), B_{i}^{\prime}(s, t)=\omega_{i}\left(v^{\prime}\right)$, and $\phi_{*}\left(v^{\prime}\right)=v$.

REMARK. If $\left(\omega_{i}^{\prime}\right)$ also defines a parallelism for $M^{\prime}$ and 2.3 is satisfied for each straight line $\sigma$ starting at $x_{0}^{\prime}$, then a similar arguments show that such a $\phi$ exists in a sufficiently small neighborhood of $x_{0}^{\prime}$. Of course, this is nothing more than Cartan's theorem that the component in a normal coordinate system of the curvature and torsion tensors of an affine connection uniquely determine the connection. We shall refer to this as the local version of Proposition 2.1.

3. The constant manifolds of $\Omega$ and the pseudogroup of automorphisms of an absolute parallelism. Let $M$ be a manifold with an absolute parallelism defined by forms $\left(\omega_{i}\right)$, with functions $c_{j k i}, c_{j k i, h}, \cdots$ defined by 1.1 and 1.2 and with $\Omega$ the ring of functions generated by these functions. For $x \in M$, let $\Omega^{x}$ be the connected component containing $x$ of $\{y \in M: f(y)=f(x)$ for all $f \in \Omega\}$. Let $M_{x}(\Omega)=\left\{v \in M_{x}: d f(v)=0\right.$ for all $\left.f \in \Omega\right\}$. It is asserted in Theorem 2.5 of [8] that $\Omega^{x}$ is a submanifold of $M$ and that $M_{x}(\Omega)$ is the tangent space to $\Omega^{x}$ at $x$. This theorem is false, as the following counter-example, given to me by I. M. Singer, shows:

Let $M$ be the plane of two real variables $s$ and $t$. Let $f(s)$ be the following $C^{\infty}$ function: $f(s)=1$ for $s \leqq 0, f(s)=e^{-1 / s^{2}}+1$ for $s \geqq 0$. Let $\omega_{1}=d s$, $\omega_{2}=f(s) d t . \omega_{1}$ and $\omega_{2}$ define an absolute parallelism for $M$. Further, $d \omega_{1}=0$, $d \omega_{2}=\left(f^{\prime}(s) / f\right) \omega_{1} \wedge \omega_{2}$. Thus $\Omega$ is generated by $f^{\prime}(s) / f(s)$ and its derivatives. But, the set of points where all elements of $\Omega$ are zero is the set of all $(s, t)$ with $s \leqq 0$, which is not a manifold.

Examining the proof of Theorem 2.5 of [8], it may be seen that the error occurs in the last paragraph, i.e., lines $21-24$ on page 308 . Thus, the proof of 2.13 of [8] is correct, and this translated into the notation used here yields the following fact:

$$
\operatorname{dim} M_{x}(\Omega)=\operatorname{dim} M_{y}(\Omega) \text { for all } y \in \Omega^{x} .
$$

$\Omega^{x}$ is called the constant set of $\Omega$ passing through $x$.

Let $A$ be the pseudogroup of local automorphisms of the parallelism. Thus, a $\phi \in A$ is a diffeomorphism between open sets $U_{d}(\phi)$ and $U_{r}(\phi)$ of $M$ such that $\phi^{*}\left(\omega_{i}\right)=\omega_{i}$. Thus also $\phi^{*}(\Omega)=\Omega$. Let $A(M)$ denote the corresponding connected group of automorphisms of the parallelism, i.e., the set of $\phi \in A$ with $U_{d}(\phi)=M=U_{r}(\phi)$, such that $\phi$ can be continuously deformed in $A$ to the identity. It is known that $A(M)$ is a Lie group [10], that a $\phi \in A$ has no fixed 
points unless it is the identity, and that $\Omega^{x}$ contains the orbit of $x \in M$ under the sub-pseudogroup of $A$ consisting of those $\phi \in \mathbf{A}$ that can be continuously deformed in $A$ into the identity.

We say that $\Omega$ is finitely generated in an open set $U$ of $M$ if there is an integer $N$ and elements $f_{1}, \cdots, f_{N}$ in $\Omega$ such that any $f \in \Omega$ can, in $U$ be written in the form $f=F\left(f_{1}, \cdots, f_{N}\right)$, where $F\left(u_{1}, \cdots, u_{N}\right)$ is a $C^{\infty}$ function of $N$ real variables. We say that $\Omega$ is locally finitely generated if each point has a neighborhood $U$ in which it is finitely generated.

THEOREM 1. With the above notations, suppose either that $\Omega$ is locally finitely generated or that $M$ and the $\omega_{i}$ are real analytic. Then,

(a) $\operatorname{dim} M_{x}(\Omega)$ is constant for $x \in M$, and defines a foliation on $M$ whose leaves are the $\Omega^{x}$. The $\Omega^{x}$ are then closed submanifolds of $M$.

(b) The pseudogroup $A$ is locally transitive on the sets $\Omega^{x}, x \in M$.

(c) If the parallelism is complete and if $M$ is simply connected, then $A(M)$ is simply transitive on the sets $\Omega^{x}, x \in M$.

Proof. A vector field $X$ on $M$ is the infinitesimal generator of a 1-parameter pseudogroup of automorphisms of the parallelism if: $X\left(\omega_{i}\right)=0$, where $X\left(\omega_{i}\right)$ denotes the Lie derivative of the form $\omega_{i}$ by the vector field $X$.

(See [8] for a fuller description of the Lie derivative operation and the laws it obeys. We will use formulas $2.1-2.8$ of [8] freely.) Let $V_{i}=\omega_{i}(X)$, i.e., the $V_{i}$ are the functions that are the components of the vector field $X$ with respect to the basis of forms $\left(\omega_{i}\right)$. The condition $X\left(\omega_{i}\right)=0$ then takes the form (using 2.4 of [8]):

$$
d V_{i}=c_{k j i} V_{j} \omega_{k}
$$

Let $\sigma:[0,1] \rightarrow M$ be a curve and let $v: t \rightarrow v(t)=X(\sigma(t)) \in M_{\sigma(t)}$ be the vector field along $\sigma$ obtained by restricting $X$ to $\sigma$. Let $v_{i}(t)=\omega_{j}(v(t))=V_{i}(\sigma(t))$ be the components of $v$. Then, 3.1 implies:

$$
\frac{d}{d t} v_{i}(t)=c_{k j i}(\sigma(t)) v_{j}(t) \omega_{k}\left(\sigma^{\prime}(t)\right)
$$

Now, forget for the moment the origin of equations 3.2. They form a system of first order linear homogeneous ordinary differential equations for the $v_{i}(t)$, with a unique solution for given initial conditions.

LEMMA 3.1. Suppose that either (a) $M$ and the elements of $\Omega$ are real analytic, or (b) $\Omega$ is locally finitely generated. Suppose that $v(t), 0 \leqq t \leqq 1$, is a vector field along the curve $\sigma$ satisfying 3.2 and such that $d f(v(0))=0$ for all $f \in \Omega_{x}$. In hypothesis (a), suppose further that the vector field $v$ is piecewise real analytic. Then, in both cases, $d f(v(t))=0$ for all $f \in \Omega$. In particular, $v(t) \in M_{\sigma(t)}(\Omega)$ for $0 \leqq t \leqq 1$, hence:

$$
\operatorname{dim} M_{\sigma(0)}(\Omega) \leqq \operatorname{dim} M_{\sigma(1)}(\Omega)
$$


Proof. Suppose that $\delta(s, t), 0 \leqq s, t \leqq 1$, is a homotopy of curves such that: $\delta(0, t)=\sigma(t), 0 \leqq t \leqq 1$, and, for each $t$, the tangent vector to the curve $s \rightarrow \delta(s, t)$ at $s=0$ is $v(t)$. Thus, $\delta(s, t)$ is a deformation of $\sigma$, and $v$ is the corresponding infinitesimal deformation. Suppose that:

$$
\delta^{*}\left(\omega_{i}=A_{i}(s, t) d t+B_{i}(s, t) d s .\right.
$$

Then, our conditions require that: $A_{i}(0, t)=\omega_{i}\left(\sigma^{\prime}(t)\right)$ and $B_{i}(0, t)=\omega_{i}(v(t))$.

From 2.5, we have

$$
\frac{\partial A_{i}}{\partial s}-\frac{\partial B_{i}}{\partial t}=\delta^{*}\left(c_{j k i}\right) A_{k} B_{j}
$$

hence:

$$
\left.\frac{\partial A_{i}}{\partial s}\right|_{s=0}=\frac{d}{d t} \omega_{i}(v(t))+c_{j k i}(\sigma(t)) \omega_{k}\left(\sigma^{\prime}(t)\right) \omega_{j}(v(t))=0
$$

using 2.2. Thus,

$$
\begin{aligned}
\frac{d}{d t}\left(d c_{j k i}(v(t))\right) & =\left.\frac{\partial}{\partial t}\left(\frac{\partial}{\partial s} c_{j k i}(\delta(s, t))\right)\right|_{s=0} \\
& =\left.\frac{\partial}{\partial s}\left(\frac{\partial}{\partial t} c_{j k i}(\delta(s, t))\right)\right|_{s=0} \\
& =\left.\frac{\partial}{\partial s}\left(c_{j k i, h}(\delta(s, t)) A_{h}\right)\right|_{s=0} \\
& =\left.\frac{\partial}{\partial s}\left(c_{j k i, h}(\delta(s, t))\right)\right|_{s=0} \omega_{h}\left(\sigma^{\prime}(t)\right),
\end{aligned}
$$

using 3.3 whence:

$$
\frac{d}{d t}\left(d c_{j k i}(v(t))\right)=d c_{j k i, h}(v(t)) \omega_{h}\left(\sigma^{\prime}(t)\right) .
$$

Continuing:

$$
\begin{aligned}
\frac{d}{d t}\left(d c_{j k i, h}(v(t))\right) & =\left.\frac{\partial}{\partial t}\left(\frac{\partial}{\partial s} c_{j k i, h}(\delta(s, t))\right)\right|_{s=0} \\
& =\left.\frac{\partial}{\partial s}\left(\frac{\partial}{\partial t} c_{j k i, h}(\delta(s, t))\right)\right|_{s=0} \\
& =\left.\frac{\partial}{\partial s}\left(c_{j k i, h, h_{1}} A_{h_{1}}\right)\right|_{s=0} \\
& =\left.\frac{\partial}{\partial s}\left(c_{j k i, h, h_{1}}\right)\right|_{s=0} \omega_{h_{1}}\left(\sigma^{\prime}(t)\right) \\
& =d c_{j k i, h, h_{1}}(v(t)) \omega_{h_{1}}\left(\sigma^{\prime}(t)\right) .
\end{aligned}
$$


The general formula is seen to be:

$$
\frac{d}{d t}\left(d c_{j k i, h, h_{1}, \ldots, h_{p}}\right)(v(t))=d c_{j k i, h, h_{1}, \ldots, h_{p+1}}(v(t)) \omega_{p+1}\left(\sigma^{\prime}(t)\right) .
$$

Thus, 3.4 gives an infinite system of linear homogeneous differential equations for the functions $d c_{j k i, h_{1}, \ldots, h_{p}}(v(t)) .1 \leqq j, k, i, h, h_{1}, \cdots, h_{p} \leqq n, 0 \leqq p<\infty$, with, by the hypotheses of Lemma 3.1, zero initial conditions. Thus, in hypothesis (a), the functions $d c_{j k i}(v(t)), d c_{j k i, h}(v(t))$, etc., are identically zero, thus proving the lemma. In case (b), proceed as follows:

Since we want to prove that the functions $d c_{j k i}(v(t)), d c_{j k i, h}(v(t))$, etc., are identically zero, it suffices to work locally, i.e., we can suppose that $\Omega$ is finitely generated on $M$. Relabel the generators of $\Omega$ as $c_{\alpha}, 1 \leqq \alpha<\infty$, with $c_{1}, \cdots, c_{N}$ such that, for $\alpha>N$, there is a function $F_{\alpha}\left(u_{1}, \cdots, u_{N}\right)$ of $N$ real variables such that: $c_{\alpha}=F_{\alpha}\left(c_{1}, \cdots, c_{N}\right)$. Then, for $\alpha>N$,

$$
d c_{\alpha}(v(t))=\sum_{\beta=1}^{N} \frac{\partial F_{\alpha}}{\partial u_{\beta}}\left(c_{1}(\sigma(t)), \cdots, c_{N}(\sigma(t))\right) d c_{\beta}(v(t)) .
$$

Thus, we see that the infinite system 3.4 is equivalent to a finite system of homogeneous linear differential equations for the functions $d c_{x}(v(t))$, with zero initial conditions. These function of $t$ must then be identically zero, whence the conclusion of Lemma 3.1 in case (b).

Now return to the proof of Theorem 1. Lemma 3.1 proves (a) since, given $x, y \in M$, we have only to join $x$ and $y$ by a curve $\sigma:[0,1] \rightarrow M$ with $\sigma(0)=x$, $\sigma(1)=y$ (and suppose, in the real analytic case, that $\sigma$ is piecewise analytic). By Lemma $3.1, \operatorname{dim} M_{\sigma(0)}(\Omega) \leqq \operatorname{dim} M_{\sigma(1)}(\Omega)$, hence by symmetry,

$$
\operatorname{dim} M_{x}(\Omega)=\operatorname{dim} M_{y}(\Omega) .
$$

Let $x \in M$ and let $f_{1}, \cdots, f_{m} \in \Omega$ be functions so that:

(i) $d f_{1}, \cdots, d f_{m}$ are linearly independent at $x$, and

(ii) $M_{x}(\Omega)=\left\{v \in M_{x}: d f_{1}(v)=\cdots=d f_{m}(v)=0\right\}$.

Then, there is a neighborhood $U$ of $x$ in which (i) and (ii) hold, by 3.5. Thus, any $f \in \Omega$ can, in $U$, be written as a function of the $f_{1}, \cdots, f_{m}$. This shows that the field of tangent planes $x \rightarrow M_{x}(\Omega)$ defines a foliation on $M$. For $x \in M$, let $L^{x}$ be the leaf of the foliation. Since $x \rightarrow M_{x}(\Omega)$ defines a (nonsingular) foliation ("involutive distribution," in Chevalley's language [4]), $L^{x}$ can be defined as:

$L^{x}=\left\{y \in M: y\right.$ can be joined to $x$ by a piecewise $C^{\infty}$ regular curve on which all the $\Omega$ are constant $\}$.

To prove that $L^{x}=\Omega^{x}$, it suffices to show that $L^{x}$ is closed in $M$, since it is clearly, by the above results, open in $\Omega^{x}$. Suppose then that $z$ is a point of $M$ lying in the closure of $\Omega^{x}$. Choose $f_{1}^{\prime}, \cdots, f_{m}^{\prime} \in \Omega$ and a neighborhood $U^{\prime}$ of $z$ such that (a) the $f_{1}^{\prime}, \cdots, f_{m}^{\prime}$ are functionally independent in $U^{\prime}$, and (b) $M_{y}(\Omega)$ $=\left\{v \in M_{y}: d f_{1}^{\prime}(v)=\cdots=d f_{m}^{\prime}(v)=0\right\}$ for $y \in U$. 
Further, $U$ can be chosen sufficiently small so that

$$
\Omega^{z} \cap U=\left\{y \in U: f_{1}^{\prime}(y)=f_{1}^{\prime}(z), \cdots, f_{m}(y)=f_{m}^{\prime}(z)\right\} .
$$

But, since $f(z)=f(x)$ for all $f \in \Omega_{1}$ since $z$ lies in the closure of $\Omega^{x}$, we must have: $\Omega^{x} \cap U$ lies in the leaf of the foliation passing through $z$, i.e., $z$ belongs to $L^{x}$.

Now we turn to the proof of (b), using the following lemma.

Lemma 3.2. Suppose that $\operatorname{dim} M_{x}(\Omega)=\operatorname{dim} M_{y}(\Omega)$ for $x, y \in M$ and that $D$ is an open simply connected subset of $M$. Then, given $x \in D, v \in M_{x}(\Omega)$, there is a unique vector field $X$ on $D$ satisfying: $X(x)=v, X\left(\omega_{i}\right)=0$, and $X(f)=0$ for all $f \in \Omega$, i.e., $X$ is an infinitesimal parallelism isomorphism that is tangent to the leaves of the foliation $x \rightarrow M_{x}(\Omega)$.

Proof. First we prove the lemma in case $D$ is sufficiently small. Consider 3.1, i.e., the conditions that a vector field $X$ satisfy $X\left(\omega_{i}\right)=0$ :

$$
d\left(\omega_{i}(X)\right)=c_{k j i} \omega_{j}(X) \omega_{k} .
$$

These conditions, together with the condition $X(f)=0$ for all $f \in \Omega$, are completely integrable, i.e., the following exterior differential system in the space $D \times$ (space of variables $\left(V_{i}\right)$ ) is completely integrable:

$$
\begin{gathered}
d V_{i}-c_{k j i} V_{j} \omega_{k}=0, \\
d f(X)=0 \text { for all } f \in \Omega, \text { i.e., } f_{i} V_{i}=0, \text { where } d f=f_{i} \omega_{i} .
\end{gathered}
$$

This proves Lemma 3.2 "locally." Now, a vector field $X$ satisfying $X\left(\omega_{i}\right)=0$ is, as we have seen, determined by its value at a point. Thus, the sheaf of germs of vector fields $X$ satisfying $X\left(\omega_{i}\right)=0$ is locally constant and the dimension of its stalk at any point $x \in M$ is $\operatorname{dim} M_{x}(\Omega)$. Thus, Lemma 3.2 now follows from standard arguments.

Statement (b) of Theorem 1 now follows immediately from Lemma 3.2. Statement (c) of Theorem 1 follows also: Let $\mathbf{A}(M)$ denote the Lie algebra of vector fields $X$ on $M$ such that $X\left(\omega_{i}\right)=0$. If $M$ is simply connected, $\operatorname{dim} \mathbf{A}(M)$ $=\operatorname{dim} M_{x}(\Omega)$, for all $x \in M$.

Lemma 3.3. If $M$ is simply connected and if the parallelism defined by $\left(\omega_{i}\right)$ is complete, then the integral curves of each $X \in \mathbf{A}(M)$ can be indefinitely extended. Let $G$ be the simply connected Lie group whose Lie algebra is $\mathbf{A}(M)$. Then, also, the action of $\mathrm{A}(M)$ on $M$ can be integrated to give rise to an action of $G$. The orbits of $G$ are precisely the sets $\Omega^{x}$, i.e., the leaves of the foliation $x \rightarrow M_{x} \Omega$.

First, we note that Lemma 3.3 proves (c) of Theorem 1. For the orbits of $G$, i.e., the orbits of $G$, are open in the $\Omega^{x}$ and closed in the $\Omega^{x}$, since the orbits of $G$ are closed in $M$ [10]. 
To prove the first part of Lemma 3.3, notice that any integral curve $\sigma:[0, a] \rightarrow M$ of $X$ is a straight line of the parallelism $\left(\omega_{i}\right)$ : hence can be indefinitely extended: For,

$$
\frac{d}{d t} \omega_{i}\left(\sigma^{\prime}(t)\right)=\frac{d}{d t} \omega_{i}(X)(\sigma(t))=X\left(\omega_{i}(X)\right)(\sigma(t))=0
$$

by formula 2.3 of [8]. The second part of Lemma 3.3 follows from the standard Lie theory of transformation groups. This completes the proof of Theorem 1.

Remarks. These facts are analogous to results of K. Nomizu [11] and I. M. Singer [13] proved in a Riemannian setting. If (c) is satisfied, then the space $M / \Omega$ of the constant submanifolds of $\Omega$ is the orbit space $A(M) \backslash M$, hence it is a manifold such that the projection map $M \rightarrow M / \Omega$ is a $C^{\infty}$ map of maximal rank whose fibres are the $\Omega^{x}$. This is also true in the more general (?) case that the holonomy group in the sense of [1] of the foliation whose leaves are the constant manifolds of $\Omega$ is the identity. The proof involves a slight variant of the proof of Theorem 4.4 of [7] and hence will be omitted.

We now turn to the proof of Theorem 2 stated in $\S 1$. Let $x_{0}^{\prime} \in M^{\prime}$ and $x_{0} \in M$ be points such that $\psi\left(x_{0}^{\prime}\right)=\pi\left(x_{0}\right)$. Let $\sigma:[0,1] \rightarrow M^{\prime}$ be a curve on $M^{\prime}$ and let $\sigma_{D}:[0,1] \rightarrow M$ be its development in $M$. We want to prove that, for each $f \in \Omega$, $\psi^{*}(f)(\sigma(t))=f\left(\sigma_{D}(t)\right), 0 \leqq t \leqq 1$, for then we can apply Proposition 2.1 to construct the desired $\phi$, which will also satisfy $\pi \phi=\psi$. This, however, is a purely local matter, i.e., if it can be proved whenever $\sigma$ is sufficiently small, it is proved for arbitrary $\sigma$ by a subdivision process.

Locally, the situation looks as follows: Let $\mathbf{f}_{1}, \cdots, \mathbf{f}_{N}$ be functions on $M / \Omega$ that form a coordinate system about $\pi\left(x_{0}\right)$, and let $f_{1}=\pi^{*}\left(\mathbf{f}_{1}\right), \cdots, f_{N}=\pi^{*}\left(\mathbf{f}_{N}\right)$. There are functions $F_{j k i}\left(u_{1}, \cdots, u_{N}\right), F_{j k i, h}\left(u_{1}, \cdots, u_{N}\right), \cdots$ of $N$ real variables such that

$$
c_{j k i}=F_{j k i}\left(f_{1}, \cdots, f_{N}\right) \text {, }
$$

$$
c_{j k i, h}=F_{j k i, h}\left(f, \cdots, f_{N}\right), \text { etc. }
$$

Further, we can suppose that the $f$ 's occur among the $c$ 's. We are given as hypothesis that

$$
c_{j k i}^{*}=F_{j k i}\left(\psi^{*}\left(\mathbf{f}_{1}\right), \cdots, \psi^{*}\left(\mathbf{f}_{N}\right)\right), \text { etc. }
$$

Using 1.1, 1.2 and 1.3 , we have

$$
\frac{d}{d t} c_{j k i}^{\prime}(\sigma(t))=c_{j k i, h}^{\prime}(\sigma(t)) \omega_{h}^{\prime}\left(\sigma^{\prime}(t)\right), \text { etc. }
$$

Combining 3.5, 3.6, 3.7 and 3.8 with the condition that $\omega_{h}^{\prime}\left(\sigma^{\prime}(t)\right)=\omega_{h}\left(\sigma_{D}^{\prime}(t)\right)$ we see that the functions 
and

$$
t \rightarrow\left(f_{1}\left(\sigma_{D}(t)\right), \cdots, f_{N}\left(\sigma_{D}(t)\right)\right.
$$

$$
t \rightarrow\left(\psi^{*}\left(f_{1}\right)(\sigma(t)), \cdots, \psi^{*}\left(f_{N}\right)(\sigma(t))\right)
$$

satisfy the same system of ordinary differential equations with the same initial conditions, hence coincide. This is what we had to prove to finish the proof of Theorem 2.

REMARK. There are generalizations of Theorem 2 that one can obtain by considering $M / \Omega$ as a "manifold with singularities," that we hope to deal with in another work. In case $\Omega$ is locally finitely generated, i.e., $M / \Omega$ is the base space of a foliation on $M$, the generalization obtained by using the "generalized manifold" structure for $M / \Omega$ customary in the theory of foliations is proved in precisely the same way.

It is obvious that completeness of the Riemannian metric $d s^{2}=\omega_{i} \cdot \omega_{i}$ implies completeness of the parallelism $\left(\omega_{i}\right)$. We may note one fairly obvious case when the converse is true:

THEOREM 3. Suppose $M$ and the parallelism $\left(\omega_{i}\right)$ on $M$ are real analytic and that the parallelism is complete. Then the Riemannian metric $d s^{2}=\omega_{i} \cdot \omega_{i}$ is complete if:

For every geodesic of the metric $\sigma:[0,1] \rightarrow M$ parametrized proportionally to arc-length the function $t \rightarrow c_{i j k}(\sigma(t))$ can be extended to real-analytic functions of $t$ over $0 \leqq t<\infty$.

Proof. If $\sigma^{*}\left(\omega_{i}\right)=A_{i}(t) d t$, it is easy to see that $\sigma$ is a geodesic if and only if:

$$
\frac{d}{d t} A_{i}(t)=c_{i j k}(\sigma(t)) A_{j}(t) A_{k}(t)
$$

Suppose that $\gamma_{i j k}(t), 0 \leqq t<\infty$, are real analytic such that $\gamma_{i j k}(t)=c_{i j k}(t)$ for $0 \leqq t \leqq 1$. Define $A_{i}(t), 0 \leqq t<\infty$, so that:

$$
\frac{d}{d t} A_{i}(t)=\gamma_{i j k}(t) A_{j}(t) A_{k}(t) \quad \text { for } 0 \leqq t<\infty,
$$

and

$$
\sigma^{*}\left(\omega_{i}\right)=A_{i}(t) d t \quad \text { for } 0 \leqq t<\infty .
$$

Of course, we must be assured that 3.10 has a solution over all of $[0, \infty)$. Trying to apply the usual continuation arguments, suppose it is defined over $[0, T)$. From 3.9 and the skew-symmetry of $\gamma_{i j k}$ in $i$ and $j$ it follows that:

$$
\frac{d}{d t}\left(A_{i}(t) A_{i}(t)\right)=0
$$

Without loss of generality, we can suppose that $A_{i}(t) A_{i}(t)=1$. Hence 


$$
\left|\frac{d}{d t} A_{i}(t)\right| \leqq \max _{j, k ; 0 \leqq t \leqq D}\left|\gamma_{i j k}(t)\right| \cdot n^{2} \quad \text { for } 0 \leqq t<T,
$$

and the curve $t \rightarrow\left(A_{i}(t)\right)$ in $R^{n}, 0 \leqq t<T$, has finite length. Then, $\lim _{i \rightarrow T} A_{i}(t)$ must exist for each $i$. This implies that the solution of 3.10 can be continued beyond $T$. Since $T$ was arbitrary, this implies that 3.10 has a solution over $0 \leqq t<\infty$. Now, define $\sigma$ over $0 \leqq t<\infty$ so that it satisfies 3.11, using completeness of the parallelism. By real-analyticity, $\sigma$ so extended is a geodesic. Then, we have proved that each piece of geodesic can be extended over $0 \leqq t<\infty$, i.e., the metric is complete. q.e.d.

4. Global reducibility. As our principal application, we discuss conditions under which global reducibility of a parallelism can be proved knowing local reducibility.

Suppose then that $M$ is a space with parallelism $\left(\omega_{i}\right)$. Choose the following range of indices and summation connections, $1 \leqq a, b, c \leqq m ; m+1 \leqq u$, $v, w, x, \cdots \leqq n$. The parallelism is said to be reducible if the basis $\left(\omega_{i}\right)$ of invariant forms can be chosen so that

$$
d \omega_{a}=c_{b c a} \omega_{b} \wedge \omega_{c}
$$

$$
d \omega_{u}=c_{v w u} \omega_{v} \wedge \omega_{w}
$$

Then, the Pfaffian system $\omega_{a}=0\left(\right.$ resp $\left.\omega_{u}=0\right)$ defines a foliation on $M$. Let $x_{0} \in M$. Let $F$ (resp $F^{\prime}$ ) be the leaf through $x_{0}$, i.e., a maximal, connected integral submanifold of the foliation $\omega_{a}=0\left(\operatorname{resp} \omega_{u}=0\right)$. Let $i: F \rightarrow M\left(\operatorname{resp} i^{\prime}: F^{\prime} \rightarrow M\right)$ be the inclusion map. Then, $\left(i^{*}\left(\omega_{u}\right)\right)$ (resp $\left.\left(i^{\prime *}\left(\omega_{a}\right)\right)\right)$ defines a parallelism on $F$ (resp $F^{\prime}$ ) that is complete if the parallelism on $M$ is complete, which we will assume.

LEMMA 4.1. If $M$ is simply connected, there is a map $\phi: M \rightarrow F$ such that

(a) $\phi i=$ identity,

(b) $\phi^{*} i^{*}\left(\omega_{u}\right)=\omega_{u}$.

Proof. We hope to apply Proposition 2.1 to prove this. Suppose then that $\sigma:[0,1] \rightarrow M$ is a $C^{\infty}$ curve with $\sigma(0)=x$. Suppose $\sigma^{*}\left(\omega_{i}\right)=A_{i}(t) d t$. Suppose $\sigma_{1}:[0,1] \rightarrow F$ is the curve such that

$$
\begin{aligned}
& \sigma_{1}(0)=x_{0}, \\
& \sigma_{1}^{*}\left(\omega_{a}\right)=0, \\
& \sigma_{1}^{*}\left(\omega_{u}\right)=A_{u}(t) d t .
\end{aligned}
$$

In order to satisfy the hypotheses of Proposition 2.1 and hence construct the map $\phi: M \rightarrow F$, we must show that: 


$$
c_{u v w}(\sigma(t))=c_{u v w}\left(\sigma_{1}(t)\right) \quad \text { for } 0 \leqq t \leqq 1 \text {, and all such } \sigma .
$$

To prove this, define a homotopy $\delta(s, t), 0 \leqq s, t \leqq 1$, such that:

$$
\begin{aligned}
& \delta(s, 0)=x_{0}, \quad 0 \leqq s \leqq 1, \\
& \delta(0, t)=\sigma(t), \quad \delta(1, t)=\sigma_{1}(t), \\
& \delta^{*}\left(\omega_{i}\right)=A_{i}(s, t) d t+B_{\imath}(s, t) d s, \text { where } \\
& A_{u}(s, t)=(1-s) A_{a}(t), 0 \leqq s, t \leqq 1, \text { and } \\
& A_{u}(s, t)=A_{u}(t) .
\end{aligned}
$$

(To define $\delta$, use the completeness of $M$. Use the fact that it is defined by $C^{\infty}$ differential equations to prove that it is $C^{\infty}$.) Using 4.1,

hence

$$
d c_{u v w}=c_{u v w, x} \omega_{x},
$$

$$
\frac{\partial}{\partial s}\left(c_{u v w}(\delta(s, t))\right)=c_{u v w, x}(\delta(s, t)) B_{x}(s, t)
$$

Using 4.1 again,

$$
\frac{\partial A_{x}}{\partial s}-\frac{\partial B_{x}}{\partial t}=c_{u v x}(\delta(s, t)) A_{v} B_{u}
$$

By 4.3, $\partial A_{x} / \partial s=0$, hence $B_{x}$, for $s$ held fixed, satisfies a system of homogeneous, linear differential equations with (by 4.3 ) initial conditions zero. Hence,

$$
\frac{\partial}{\partial s}\left(c_{u v w}(\delta(s, t))\right)=0 \text { or } c_{u v w}(\delta(0, t))=c_{u v w}(\delta(1, t)) .
$$

This proves 4.2, hence provesıLmma 4.1 .

THEOREM 4. If $M$ is simply connected and has a complete parallelism $\left(\omega_{i}\right)$ satisfying 4.1 , then the parallelism on $M$ is isomorphic to the product parallelism on $F \times F^{\prime}$.

Proof. By Lemma 4.1, construct maps $\phi^{\prime}: M^{\prime} \rightarrow F^{\prime}$ and $\phi: M \rightarrow F$. Since $\phi i=$ identity and $\phi^{\prime} i^{\prime}=$ identity, $F$ and $F^{\prime}$ are simply connected, as is $F \times F^{\prime}$ then. Construct the product map $\phi^{\prime \prime}: M \rightarrow F \times F^{\prime}$. It is clear that it is a parallelism homomorphism, and a local diffeomorphism. The proof will be finished on proving:

LEMMA 4.2. Suppose $M$ and $M^{\prime}$ are spaces with absolute parallelisms, $M$ complete and $M^{\prime}$ simply connected. Let $\phi: M \rightarrow M^{\prime}$ be a parallelism homomorphism that is a local diffeomorphism. Then, $\phi$ is a diffeomorphism of $M$ with $M^{\prime}$. 
Proof. Suppose the forms $\left(\omega_{i}\right)$ (resp $\left(\omega_{i}^{\prime}\right)$ ) define the parallelism on $M$ (resp $\left.M^{\prime}\right) . \phi: M \rightarrow M^{\prime}$ is a parallelism homomorphism if: $\phi^{*}\left(\omega_{i}^{\prime}\right)=A_{i j} \omega_{j}$, with $\left(A_{i j}\right)$ a constant matrix. Since we are requiring that $\phi$ be a local diffeomorphism, it must be a nonsingular matrix. Let $\sigma_{1}:[0,1] \rightarrow M^{\prime}$ be a curve, with $\sigma_{1}^{*}\left(\omega_{i}^{\prime}\right)=A_{i}(t) d t$. By well-known arguments [1, Theorem A, p. 360], it suffices to prove that, for each $x_{0} \in \phi^{-1}\left(\sigma_{1}(0)\right)$, there is a curve $\sigma_{1}:[0,1] \rightarrow M$ with $\sigma(0)=x_{0}$ and $\phi(\sigma(t))=\sigma_{1}(t)$. Then, if $\sigma^{*}\left(\omega_{i}\right)=B_{i}(t)$, we must have

$$
A_{i}(t)=A_{i j} B_{j}(t)
$$

Conversely, $B_{i}(t)$ can be obtained by solving these linear equations, $\sigma$ can be defined using completeness of the parallelism on $M$, and then $\phi \sigma=\sigma_{1}$ by the uniqueness part of 2.1 .

5. Remarks on the proof of the de Rham reducibility theorem [5], using Theorem 4. Let $B$ be a Riemannian manifold of dimension $n, 1 \leqq i, j, \cdots \leqq n$. Let $E$ be a bundle of orthonormal frames over $B$, a manifold with an absolute parallelism $\left(\omega_{i}, \omega_{i j}\right)$ defined by the Levi-Civita parallelism on $B$, satisfying the structure equations $[1 ; 3]$ :

$$
\begin{aligned}
& d \omega_{i}=\omega_{i j} \wedge \omega_{j}, \quad \omega_{i j}+\omega_{i j}=0, \\
& d \omega_{i j}=\omega_{i k} \wedge \omega_{k j}-R_{i j k l} \omega_{k} \wedge \omega_{l} .
\end{aligned}
$$

To say that the holonomy group of $B$ is reducible is (more or less) to say that the structure group of $E$ can be reduced from $O(n)$ to $O(m) \times O(n-m)$ in a torsionfree way. For us, this will mean that there is a submanifold $E^{\prime}$ of frames such that:

$$
\begin{aligned}
\omega_{a u} & =0 \text { on } E^{\prime}, \quad(1 \leqq a, b, \cdots \leqq m ; m+1 \leqq u, v, \cdots \leqq n) . \\
d \omega_{u v} & =\omega_{u w} \wedge \omega_{w v}-R_{u v w x} \omega_{w} \wedge \omega_{x}, \\
d \omega_{a b} & =\omega_{a c} \wedge \omega_{c b}-R_{a b c d} \omega_{c} \wedge \omega_{d} .
\end{aligned}
$$

We indicate how this is equivalent to the usual definition of reducibility. Pick a $b_{0} \in B$ and subspaces $T, T^{\prime} \subset B_{b_{0}}$ such that $B_{b_{0}}=T \oplus T^{\prime}, \operatorname{dim} T=m$, $\operatorname{dim} T^{\prime}=n-m$, and $T$ and $T^{\prime}$ are invariant under the holonomy group, i.e., parallel translation of a vector of $T\left(\operatorname{resp} T^{\prime}\right)$ around a loop beginning at $x_{0}$ gives a vector in $T$ (resp $T^{\prime}$ ). Let $e \in E$ be an orthonormal basis of $B_{b_{0}}$ whose first $m$ vectors be in $T$, last $n-m$ in $T^{\prime} . E^{\prime}$ is then the set of bases of the tangent space of $B$ obtained by parallel translating $e$ along all curves in $B$ starting at $b_{0}$. Thus, we get perpendicular fields $b \rightarrow T_{b}$ and $b \rightarrow T_{b}^{\prime}$ of tangent subspaces of $B$ obtained by parallel translating $T$ and $T$ 'along any curve joining $b_{0}$ to $b$. It can be proved that these fields are completely integrable, i.e., define two foliations on $B$, and that $R\left(v_{1}, v_{2}\right)=0$ for $v_{1} \in T_{b}, v_{2} \in T_{b}$,' all $b \in B$, where $R(),($ ) is the Riemann curvature tensor. 
After making explicit the relation between parallel translation and $R()()$, on $B$ and the forms $\omega_{i}, \omega_{i j}, R_{i j k i} \omega_{k} \wedge \omega_{l}$ on $E$ [1], relations 5.1 are obtained.

Suppose the metric on $B$ is complete and that $B$ is simply connected. It is easy to see that $\left(\omega_{i}, \omega_{a b}, \omega_{u v}\right)$ defines a complete parallelism on $E^{\prime}$. (In fact, the metric $d \theta^{2}=\omega_{\imath} \cdot \omega_{i}+\omega_{a b} \cdot \omega_{a b}+\omega_{u v} \cdot \omega_{u v}$ is complete.) However, $E^{\prime}$ is not necessarily simply connected. To get around this, let $M$ be the simply connected covering space of $E^{\prime}$. The forms pull up to $M$ to define a complete parallelism for $M$ again satisfying 5.1. (We denote the forms pulled up to $M$ by the same letters.) The map $M \rightarrow B$ is a fibre map, hence its fibres are connected, since $B$ is simply connected. The fibres are the leaves of the foliation $\omega_{i}=0$. By Theorem 5, $M$ is isomorphic to the product of the leaves $F$ and $F^{\prime}$ of the foliation defined by: $\omega_{a}=0=\omega_{a b}$ and $\omega_{u}=0=\omega_{u v}$. It is easy to see that the image of $F$ (resp. $F^{\prime}$ ) is a leaf $L$ (resp $L^{\prime}$ ) in $B$ of the folati on $b \rightarrow T_{b}$ (resp $b \rightarrow T_{b}^{\prime}$ ). The isomorphism $F \times F^{\prime} \rightarrow M$ constructed by Theorem 4 then passes to the quotient to define a mapping $L \times L^{\prime} \rightarrow B$ that can be shown to be a local isometry. Theorem A,p. 360 of [1], then proves that this map is an isometry.

\section{BIBLIOGRAPHY}

1. W. Ambrose, Parallel translation of Riemannian curvature, Ann. of Math. (2) 64 (1956), 337-363.

2. E. Cartan, Oeuvres complètes, Gauthier-Villars, Paris, 1955.

3. - Géometrie des éspaces de Riemann, Gauthier-Villars, Paris, 1946.

4. C. Chevalley, Lie groups. I, Princeton Univ. Press, Princeton, N. J., 1946.

5. G. de Rham, Sur la réductibilité d'un éspace de Riemann, Comment. Math. Helv. 26 (1952), 328-344.

6. C. Ehresmann, Les connexions infinitésimals dans un éspace fibré différentiable, Colloque de topologie, Brussels, 1950, pp. 29-55. 445-457.

7. R. Hermann, On the differential geometry of foliations, Ann. of Math. (2) 72 (1960),

8. - The differential geometry of foliations. II, J. Math. Mech. 11 (1962), 303-316.

9. N. Hicks, $A$ theorem on affine connections, Illinois J. Math. 3 (1959), 242-254.

10. S. Kobayashi, Les groupes des transformations qui laissent invariant une parallélisme, Colloque de topologie de Strasbourg, 1954-1955.

11. K. Nomizu, On local and global existence of Killing vector fields, Ann of Math. (2) 72 (1960), 105-120.

12. B. Reinhart, Foliated manifolds with bundle-like metrics, Ann. of Math. (2) 69 (1959), 119-131.

13. I. M. Singer, Infinitesimally homogeneous spaces, Comm. Pure Appl. Math. 13 (1960), 685-697.

14. N. Steenrod, Topology of fibre bundles, Princeton Univ. Press, Princeton, N.J., 1951.

LINCOLN LABORATORY, LeXINGTON, MASSACHUSETTS 